\title{
Avaliação da postura corporal em mulheres com câncer de mama
}

\author{
Evalution of body posture in women with breast cancer
}

Palavras-chave

Neoplasias da mama

Fotogrametria

Postura

Biomecânica

Keywords

Breast neoplasms

Photogrammetry

Posture

Biomechanics
Correspondêncio

Maria Helena da Costa Amorim

Departamento de Enfermagem

Universidade Federal do Espirito Santo Avenida Marechal Campos, 1.468 - Maruípe

(EP: 29043-900

Vitória (ES), Brasil

Recebido

$30 / 10 / 2012$

Aceito com modificacōes

23/04/2013

\section{Resumo}

OBJETIVO: Avaliar a postura corporal de mulheres submetidas ao tratamento por câncer de mama, identificar as alterações posturais nos três primeiros meses após a cirurgia e investigar a correlação desses achados com a idade da mulher, o tipo e o lado da cirurgia. MÉTODOS: Estudo longitudinal que acompanhou a evolução postural de 39 mulheres submetidas à mastectomia e quadrantectomia. A avaliação postural foi realizada nas vistas anterior e posterior com uso da biofotogrametria antes da cirurgia, após a retirada do dreno e três meses depois da cirurgia. A análise estatística foi realizada através de testes paramétricos e não paramétricos, considerando nível de significância $p<0,05$. RESULTADOS: A média de idade das mulheres foi 50 $\pm 10,5$ anos; $48,8 \%$ foram tratadas com mastectomia sendo que em 61,5\% a neoplasia era na mama esquerda. Não houve diferença significante nos ângulos posturais medidos nas mulheres no período estudado. Porém, a pelve e o tronco das mulheres tratadas com quadrantectomia mostraram mais alinhamento $\left(90^{\circ}\right)$ quando comparados aos das mastectomizadas $\left(91,3^{\circ}\right)$; mulheres com cirurgia na mama esquerda apresentaram elevação do ombro e inclinação do tronco homolateral à cirurgia em curto prazo. CONCLUSÃO: As alterações posturais apresentaram correlação com o tipo e o lado da cirurgia. $\bigcirc$ seguimento desse grupo, após término do tratamento, é necessário para esclarecer alterações posturais a longo prazo.

\section{Abstract}

PURPOSE: To evaluate the body posture of women submitted to treatment for breast cancer, to identify the postural changes in the first three months after surgery and to investigate the correlation of these findings with the woman's age and type and side of surgery. METHODS: A longitudinal study that monitored the postural changes of 39 women who underwent mastectomy and quadrantectomy. Postural evaluation was performed using the technique of biophotogrammetry before surgery, after drain removal and three months after surgery. Statistical analysis was performed using parametric and nonparametric tests, with the level of significance set at $p<0.05$. RESULTS: The average age of the women studied was $50 \pm 10.5$ years, $48.8 \%$ underwent mastectomy and the left breast was operated in $61.5 \%$ of them. There was no statistically significant difference in the women's posture during the study period. However, the pelvis and trunk of women submitted to quadrantectomy showed better alignment $\left(90^{\circ}\right)$ compared to the women submitted to mastectomy $\left(91.3^{\circ}\right.$. The women submitted to surgery in the left breast had shoulder elevation and ipsilateral inclination of the trunk within a short period of time. CONCLUSION: Postural changes were correlated with the type and side of surgery. The follow-up of this group after completion of treatment is needed to clarify long-term postural changes.

\section{Trabalho realizado no Programa de Reabilitação em Mulheres Mastectomizadas do Hospital Santa Rita de Cássia - PREMMA -} Vitória (ES), Brasil.

'Programa de Pós-graduação em Saúde Coletiva, Universidade Federal do Espírito Santo - UFES - Vitória (ES), Brasil.

Conflito de interesse: não há. 


\section{Introdução}

O câncer de mama é o tumor mais comum entre mulheres, apresentando alta taxa de incidência e mortalidade. Dados recentes demonstram uma taxa crescente da mortalidade por câncer de mama nos últimos 27 anos no Brasil ${ }^{1}$. Para o ano de 2012, são estimados 52.680 novos casos, incidência de 52/100.000 mulheres $^{1,2}$. O tratamento depende do estadiamento clínico e histológico da doença, sendo ele realizado com cirurgia como a mastectomia, mastectomia com reconstrução imediata, quadrantectomia e tumorectomia, acompanhadas ou não do tratamento adjuvante de quimioterapia, radioterapia e hormonioterapia ${ }^{2,3}$. Algumas complicações físicas e funcionais podem ocorrer como consequência do tratamento, destacando-se: alteração de sensibilidade local, dor em membro superior homolateral à cirurgia e na cicatriz, peso e edema no membro homolateral à cirurgia, linfedema, aderência cicatricial, diminuição do movimento do ombro homolateral e alterações na postura corporal ${ }^{4-8}$.

A falta do peso da mama e as complicações do tratamento alteram a biomecânica postural. Essas alterações, se perpetuadas e não tratadas em tempo, podem causar deformidades irreversíveis ${ }^{7,9}$. Anteriorização da cabeça, protrusão de ombro, acentuação das curvas da coluna (lordose e cifose), elevação do ombro e escápula para o lado operado, rotação da pelve, inclinação da cabeça para o lado contralateral à cirurgia são alterações posturais frequentemente observadas nas mulheres em tratamento por câncer de mama ${ }^{7,9,10}$.

O objetivo deste estudo foi avaliar a postura corporal de mulheres submetidas ao tratamento por câncer de mama, identificar possíveis alterações posturais nos três primeiros meses de tratamento e correlacionar esses achados com a idade da mulher, o tipo e o lado da cirurgia.

\section{Métodos}

Este estudo foi aprovado pelo Centro de Estudo e Pesquisa do Hospital Santa Rita de Cássia (HSRC) e Comitê de Ética e Pesquisa do Centro de Ciências da Saúde da Universidade Federal do Espírito Santo (UFES), atendendo as normas da resolução 196/96 do CNS, sob o número 230/10.

Trata-se de um estudo observacional longitudinal, realizado no Ambulatório Ylza Bianco do Hospital Santa Rita de Cássia em Vitória (ES), de março a dezembro de 2011. Foram incluídas no estudo mulheres com diagnóstico de câncer de mama tratadas com mastectomia ou quadrantectomia no HSRC e, após orientações sobre a pesquisa, consentiram em participar do estudo, assinando o termo de consentimento livre e esclarecido. Foram excluídas mulheres com diagnóstico de câncer de mama recidivante e aquelas reoperadas antes de três meses após a primeira cirurgia.

Calculou-se o tamanho da amostra utilizando o programa Bioestat (5.0) e a fórmula para diferenças entre médias pareadas. Esse cálculo baseou-se nas variáveis: nivelamento de escápula e ombro, alinhamento de coluna e rotação escapular, segundo estudo transversal de Rostkowska, Bak e Samborski ${ }^{7}$. Esse estudo avaliou a postura corporal de 85 mulheres mastectomizadas encontrando alterações como: inclinação lateral de tronco, assimetria nas escápulas e na altura dos ombros, rotação da pelve e aumento das curvaturas da coluna.

As variáveis do presente estudo foram: a idade, o tipo de cirurgia, o lado do corpo onde a cirurgia foi realizada, dor na coluna, atividade ocupacional, tratamento adjuvante, complicações pós-cirúrgicas, queixa principal após três meses de cirurgia e postura corporal.

A variável dependente foi a postura corporal, avaliada em ângulos através da técnica de biofotogrametria, tendo sido analisadas as correlações com a idade, o tipo de cirurgia e o lado do corpo onde a cirurgia foi realizada. As imagens foram adquiridas com emprego de uma máquina fotográfica semiprofissional da marca Panasonic, posicionada sobre um tripé nivelado a $1 \mathrm{~m}$ do chão, com as voluntárias em trajes apropriados para avaliação postural, posicionadas sobre uma marca desenhada no chão, com pés paralelos, a uma distância de 2,2 $\mathrm{m}$ da câmera fotográfica nas vistas anterior e posterior. O processamento dessas imagens foi realizado com o Software CorelDraw ${ }^{\circledR}$ (12), sempre pelo mesmo examinador. As mulheres incluídas no estudo foram fotografadas em três momentos distintos. A primeira imagem foi registrada antes da cirurgia (momento PRÉ), a segunda registrada logo após a retirada do dreno, aproximadamente 15 a 20 dias depois da cirurgia (momento PO1) e a terceira, três meses após a cirurgia (momento PO2). Antes da aquisição das imagens, as mulheres foram mapeadas em pontos anatômicos específicos com base no protocolo de posicionamento de marcadores de Ricieri, Costa e Rosário Filho ${ }^{11}$. O mapeamento foi realizado com marcadores autoadesivos da cor branca, com $13 \mathrm{~mm}$ de diâmetro, que serviram de guia para o processamento da imagem e o traçado dos ângulos estudados. $\mathrm{Na}$ vista anterior foram marcados: glabela, incisura jugular, acrômio direito e esquerdo, cicatriz umbilical, espinha ilíaca ântero-superior direita e esquerda. Na vista posterior: processos espinhosos das vértebras C7, T9 e L5, ângulos superiores e inferiores das escápulas direita e esquerda.

No programa Suite CorelDraw ${ }^{\circledR}(12)$, as imagens foram processadas quanto à calibração dos eixos e medidas dos angulares e lineares. $\mathrm{O}$ alinhamento dos sistemas de coordenadas matriciais da imagem com o sistema de coordenadas do programa CorelDraw ${ }^{\circledR}(12)$, denominado 
de calibração das imagens, foi um procedimento inicial, e a seguir alguns ângulos foram construídos na imagem. Os ângulos de nivelamento têm como objetivo medir a relação entre um vetor que conecta dois pontos bilaterais contra um vetor ortogonal ao solo. São eles: Nivelamento de Ombro (NO), de Pelve (NP) e de Escápula (NE). Os ângulos de alinhamento têm por objetivo medir a relação entre uma linha que une dois pontos ímpares e medianos, paralelos à linha gravitacional, e uma linha paralela ao solo. São eles: Alinhamento de Coluna Superior (CS) e Inferior (CI), Alinhamento do Tronco (AT) e de CabeçaTronco (CT). Na rotação escapular direita e esquerda também se aplica o princípio do alinhamento, pois se verifica a relação entre o ângulo superior e inferior da mesma escápula (pontos ímpares) com uma linha paralela ao solo. A Distância do Talhe (DT) direito e esquerdo é uma medida linear realizada na vista posterior, para a qual são utilizadas medidas geométricas e não anatômicas. É medida pela distância linear no nível da distância média das referências marcadas em T9 e L5 (Figura 1).

A análise estatística dos resultados angulares obtidos foi realizada pelo programa Statistical Package for the Social Sciences (SPSS 18), considerando o nível de significância $\mathrm{p}<0,05$. A estatística descritiva dos resultados obtidos para os ângulos medidos nas vistas anterior e posterior e a Diferença do Talhe foi representada pela média e desvio-padrão da média.

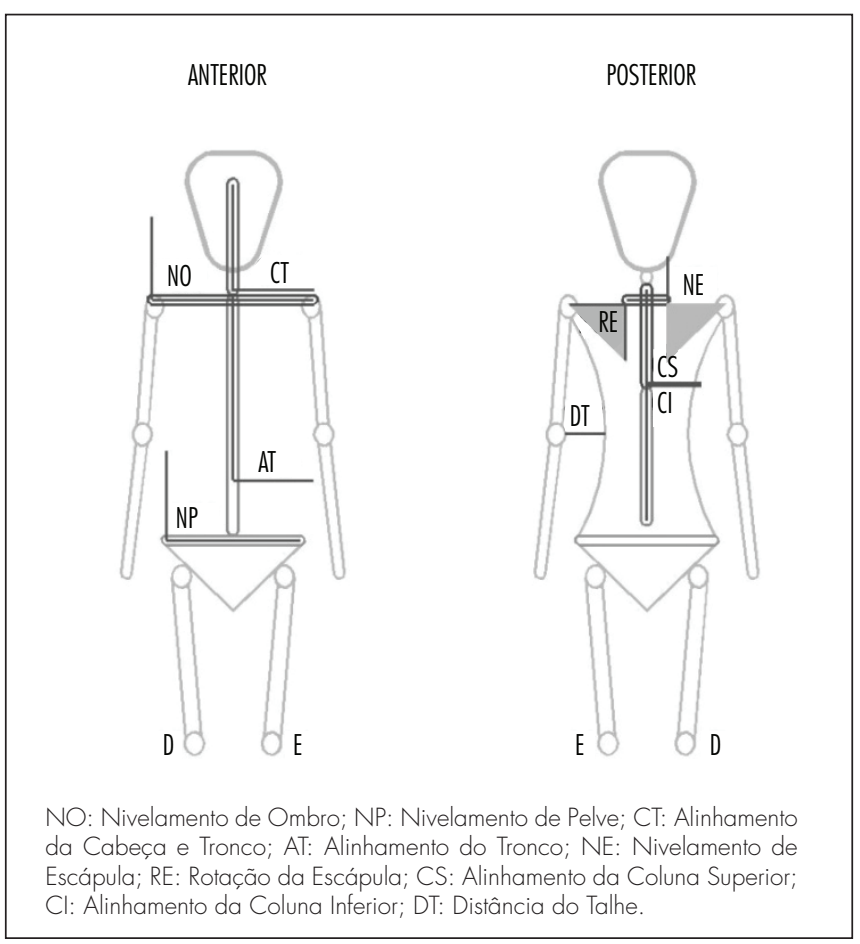

Figura 1. Ilustração dos ângulos de nivelamento e alinhamento aferidos nas vistas anterior e posterior.
O Teste de Wilcoxon foi usado para analisar a significância das modificações posturais ocorridas nos ângulos medidos nas vistas anterior e posterior nos três momentos avaliados (PRÉ, PO1 e PO2). Para verificar a correlação das modificações posturais com as variáveis — idade, tipo de cirurgia e lado da cirurgia - , utilizou-se o Teste $t$ de Student. A medida linear DT apresentou comportamento não gaussiano na análise, portanto sua correlação foi analisada pelo teste não paramétrico de Mann Whitney.

\section{Resultados}

Participaram do estudo 39 mulheres em tratamento de câncer de mama com média de idade de 50,0 $\pm 10,5$ anos variando de 25 a 71 anos. Dessas mulheres, 20 $(51,2 \%)$ haviam sido submetidas à quadrantectomia e 19 $(48,8 \%)$ à mastectomia, sendo $24(61,5 \%)$ dessas cirurgias na mama esquerda e $15(38,5 \%)$ na mama direita. Ao final da coleta de dados, $9(23 \%)$ mulheres estavam em tratamento com radioterapia e $23(58,9 \%)$ com quimioterapia e nenhuma fazia uso de prótese externa. Poucas complicações pós-cirúrgicas foram encontradas nesse grupo. Linfedema grau I, presença de seroma e deiscência cicatricial foram observados em duas mulheres $(5,1 \%)$. Quando questionadas sobre dor frequente na coluna antes da cirurgia, $24(61,5 \%)$ responderam sim, mas ao final da coleta de dados, 3 meses após a cirurgia, apenas 10 $(25,6 \%)$ relatavam dor frequente na coluna. A atividade ocupacional mais encontrada foi a do lar, exercida por 20 mulheres $(51,2 \%)$. As principais queixas relatadas pelas mulheres ao final da coleta foram: dor, edema e limitação da amplitude de movimento do membro superior homolateral à cirurgia em 8 mulheres $(20,5 \%)$, efeitos colaterais da quimioterapia em 6 mulheres $(15,4 \%)$ e parestesia na região da cicatriz, axila e braço homolateral à cirurgia em 5 mulheres $(12,8 \%)$.

Para interpretação funcional dos ângulos medidos, consideram-se valores próximos da ortogonal ou de $90^{\circ}$ como normalidade postural geométrica. Nos ângulos de NO, NP e NE, resultados inferiores a $90^{\circ}$ indicam que a referência anatômica à esquerda encontra-se mais elevada do que a mesma referência do lado direito e o contrário se aplica para valores acima de $90^{\circ}$. Para interpretação funcional dos ângulos de alinhamento da cabeça, AT , das CS (marcações em C7 e T9) e CI (marcações em T9 e L5), resultados inferiores a $90^{\circ}$ revelaram inclinação à direita, e resultados superiores a $90^{\circ}$, inclinação à esquerda, dos respectivos segmentos. A análise dos dados não mostrou diferença significante entre os ângulos medidos e a DT nos três momentos investigados.

Comparando a diferença de médias entre os tipos de cirurgia analisados (quadrantectomia e mastectomia) e os ângulos medidos, encontra-se significância para o ângulo 
Tabela 1. Diferenças de médias entre os ângulos medidos, tipos de cirurgia realizada e o lado do corpo no qual foi realizada a cirurgia

\begin{tabular}{|c|c|c|c|c|c|}
\hline Ângulos & $\begin{array}{c}\text { Cirurgia/ } \\
\text { Lado }\end{array}$ & n & Média & $\begin{array}{l}\text { Desvio- } \\
\text { padrão }\end{array}$ & Valor $p$ \\
\hline \multicolumn{6}{|c|}{ Vista anterior } \\
\hline \multirow{4}{*}{ Nivelamento de Ombro - PRÉ } & M & 19 & $89,1^{\circ}$ & $1,8^{\circ}$ & 0,7 \\
\hline & $Q$ & 20 & $89,4^{\circ}$ & $2,7^{\circ}$ & \\
\hline & $\mathrm{E}$ & 24 & $88,8^{\circ}$ & $2,3^{\circ}$ & 0,1 \\
\hline & D & 15 & $90,1^{\circ}$ & $2,2^{\circ}$ & \\
\hline \multirow{4}{*}{$\begin{array}{l}\text { Nivelamento de Ombro - } \\
\text { P01 }\end{array}$} & M & 19 & $89,3^{\circ}$ & $2,3^{\circ}$ & 0,2 \\
\hline & $Q$ & 20 & $88,3^{\circ}$ & $2,2^{\circ}$ & \\
\hline & $\mathrm{E}$ & 24 & $88,2^{\circ}$ & $2,2^{\circ}$ & 0,01 \\
\hline & D & 15 & $89,9^{\circ}$ & $2,1^{\circ}$ & \\
\hline \multirow{4}{*}{$\begin{array}{l}\text { Nivelamento de Ombro - } \\
\text { P02 }\end{array}$} & M & 19 & $89,1^{\circ}$ & $1,7^{\circ}$ & 0,5 \\
\hline & Q & 20 & $89,4^{\circ}$ & $2,3^{\circ}$ & \\
\hline & $E$ & 24 & $88,7^{\circ}$ & $1,7^{\circ}$ & 0,01 \\
\hline & D & 15 & $90,3^{\circ}$ & $2,3^{\circ}$ & \\
\hline \multirow{4}{*}{ Nivelamento de Pelve - PRÉ } & M & 19 & $91,3^{\circ}$ & $1,6^{\circ}$ & 0,04 \\
\hline & $Q$ & 20 & $89,9^{\circ}$ & $2,4^{\circ}$ & \\
\hline & E & 24 & $90,9^{\circ}$ & $2,3^{\circ}$ & 0,307 \\
\hline & D & 15 & $90,2^{\circ}$ & $2,0^{\circ}$ & \\
\hline \multirow{4}{*}{ Nivelamento de Pelve - P01 } & M & 19 & $91,1^{\circ}$ & $1,9^{\circ}$ & 0,03 \\
\hline & $Q$ & 20 & $90,0^{\circ}$ & $1,3^{\circ}$ & \\
\hline & $\mathrm{E}$ & 24 & $90,5^{\circ}$ & $1,5^{\circ}$ & 0,7 \\
\hline & D & 15 & $90,7^{\circ}$ & $2,1^{\circ}$ & \\
\hline \multirow{4}{*}{ Nivelamento de Pelve - P02 } & M & 19 & $91,4^{\circ}$ & $2,2^{\circ}$ & 0,06 \\
\hline & $Q$ & 20 & $90,2^{\circ}$ & $1,3^{\circ}$ & \\
\hline & $\mathrm{E}$ & 24 & $90,8^{\circ}$ & $1,8^{\circ}$ & 0,7 \\
\hline & D & 15 & $90,9^{\circ}$ & $2,2^{\circ}$ & \\
\hline \multirow{4}{*}{ Alinhamento Onfálico - PRÉ } & M & 19 & $89,4^{\circ}$ & $2,2^{\circ}$ & 0,05 \\
\hline & $Q$ & 20 & $90,6^{\circ}$ & $1,6^{\circ}$ & \\
\hline & E & 24 & $89,9^{\circ}$ & $2,2^{\circ}$ & 0,6 \\
\hline & D & 15 & $90,3^{\circ}$ & $1,7^{\circ}$ & \\
\hline \multirow{4}{*}{ Alinhamento Onfálico - P01 } & M & 19 & $90,0^{\circ}$ & $2,9^{\circ}$ & 0,6 \\
\hline & $Q$ & 20 & $90,4^{\circ}$ & $2,2^{\circ}$ & \\
\hline & $E$ & 24 & $90,3^{\circ}$ & $3,0^{\circ}$ & 0,9 \\
\hline & D & 15 & $90,3^{\circ}$ & $1,7^{\circ}$ & \\
\hline \multirow{4}{*}{ Alinhamento Onfálico - P02 } & M & 19 & $88,9^{\circ}$ & $2,5^{\circ}$ & 0,02 \\
\hline & $Q$ & 20 & $90,6^{\circ}$ & $2,1^{\circ}$ & \\
\hline & $\mathrm{E}$ & 24 & $90,1^{\circ}$ & $2,3^{\circ}$ & 0,3 \\
\hline & D & 15 & $89,3^{\circ}$ & $2,6^{\circ}$ & \\
\hline \multicolumn{6}{|c|}{ Vista posterior } \\
\hline \multirow{4}{*}{$\begin{array}{l}\text { Diferença da Distância do } \\
\text { Talhe - PRÉ* }\end{array}$} & $M$ & 19 & $-0,2 \mathrm{~mm}$ & $0,4 \mathrm{~mm}$ & 0,2 \\
\hline & $Q$ & 20 & $0,0 \mathrm{~mm}$ & $0,8 \mathrm{~mm}$ & \\
\hline & $\mathrm{E}$ & 24 & $-0,3 \mathrm{~mm}$ & $0,6 \mathrm{~mm}$ & 0,002 \\
\hline & $D$ & 15 & $0,3 \mathrm{~mm}$ & $0,6 \mathrm{~mm}$ & \\
\hline \multirow{4}{*}{$\begin{array}{l}\text { Diferença da Distância do } \\
\text { Talhe - P01* }\end{array}$} & M & 19 & $-0,4 \mathrm{~mm}$ & $1,0 \mathrm{~mm}$ & 0,8 \\
\hline & $Q$ & 20 & $-0,2 \mathrm{~mm}$ & $1,1 \mathrm{~mm}$ & \\
\hline & $E$ & 24 & $-0,7 \mathrm{~mm}$ & $1,1 \mathrm{~mm}$ & 0,01 \\
\hline & D & 15 & $0,2 \mathrm{~mm}$ & $0,9 \mathrm{~mm}$ & \\
\hline \multirow{4}{*}{$\begin{array}{l}\text { Diferença da Distância do } \\
\text { Talhe - P02* }\end{array}$} & M & 19 & $-0,3 \mathrm{~mm}$ & $1,0 \mathrm{~mm}$ & 0,2 \\
\hline & $Q$ & 20 & $0,1 \mathrm{~mm}$ & $1,0 \mathrm{~mm}$ & \\
\hline & $E$ & 24 & $-0,4 \mathrm{~mm}$ & $1,2 \mathrm{~mm}$ & 0,04 \\
\hline & D & 15 & $0,3 \mathrm{~mm}$ & $0,9 \mathrm{~mm}$ & \\
\hline
\end{tabular}

PRÉ: antes da cirurgia; PO 1: pós-operatório 1; PO2: pós-operatório 2; M: mastectomia; Q: quadrantectomia; E: esquerdo; D: direito. *Teste tStudent, Teste de Mann Whitney. de NP antes da cirurgia $(p=0,04)$ e no PO1 $(p=0,03)$. Observa-se que, antes da cirurgia, mulheres submetidas à mastectomia apresentavam a pelve direita mais elevada $\left(91,3^{\circ}\right)$, o que se manteve no PO1 $\left(91,1^{\circ}\right)$. Igualmente as mulheres submetidas à quadrantectomia, nas quais a pelve estava alinhada antes da cirurgia $\left(89,9^{\circ}\right)$ se mantendo alinhada no PO1 $\left(90^{\circ}\right)$ (Tabela 1). Isso indica que não houve mudança na postura de NP 15 dias após a cirurgia.

No momento PO2, o ângulo de AT apresentou variação de medidas significantes $(\mathrm{p}=0,02)$. Mulheres mastectomizadas apresentaram inclinação sutil do tronco para o lado esquerdo $\left(88,9^{\circ}\right)$, e naquelas com quadrantectomia, o tronco mostrou melhor alinhamento postural $\left(90,6^{\circ}\right)$ (Tabela 1), sugerindo uma assimetria de tronco 3 meses após a mastectomia.

Ao comparar as alterações posturais com o lado homolateral e contralateral à cirurgia, o ângulo de NO e a diferença da DT apresentaram valores significantes. $\mathrm{O}$ ombro esquerdo apresenta-se mais elevado nas mulheres com cirurgia na mama esquerda nos dois momentos avaliados após a cirurgia, PO1 88, $2^{\circ}(\mathrm{p}=0,01)$ e PO2 $88,7^{\circ}(\mathrm{p}=0,01)$. Nas mulheres submetidas à cirurgia na mama direita, o ombro apresentou leve inclinação à direita 3 meses após a cirurgia com ângulo de NO medindo $90,3^{\circ}(\mathrm{p}=0,01)$, indicando o impacto da cirurgia sobre o NO, com tendência de elevação deste para o lado da cirurgia. (Tabela 1).

$\mathrm{Na}$ medida diferença da DT, em que se avalia a inclinação lateral do tronco e/ou cintura escapular, as mulheres submetidas à cirurgia na mama esquerda apresentaram inclinação do tronco para a esquerda nos três momentos, sendo mais acentuada no PO1. Enquanto em mulheres submetidas à cirurgia na mama direita, a inclinação permaneceu para o lado direito também nos três momentos, indicando uma tendência de inclinação do tronco e/ou cintura escapular para o lado homolateral à cirurgia.

Não houve modificações significantes para os ângulos medidos e DT quando correlacionados com a idade das mulheres.

\section{Discussão}

A manutenção da postura corporal equilibrada é uma função complexa que depende da interação do sistema neural, por meio de estruturas exteroceptivas e proprioceptivas, e do sistema musculoesquelético. Após a cirurgia por câncer de mama, as mulheres adotam posturas antálgicas compensatórias, a fim de evitar dores e esconder a falta da mama. Na tentativa de se reequilibrar, a mulher altera sua biomecânica postural apresentando com frequência contraturas na região cervical e cintura escapular, elevando o ombro e escápula, e abduzindo escápula homolateral à cirurgia ${ }^{7,9}$. 
O presente estudo analisou a postura corporal de mulheres em tratamento por câncer de mama através da biofotogrametria. Essa técnica tem sido utilizada para avaliação e diagnóstico físico-funcional em diferentes áreas e especialidades. Ela propõe uma análise acessível à prática clínica, de baixo custo operacional e boa portabilidade, em que as imagens podem ser adquiridas em ambientes típicos de atenção à saúde por câmeras digitais e analisadas por programas de computador de livre acesso comercial. No presente estudo, utilizou-se a medida de oito ângulos posturais para verificar possíveis alterações na postura das mulheres avaliadas ${ }^{11-13}$.

Ao comparar os ângulos posturais medidos, não foi encontrada diferença significante antes da cirurgia, 15 dias e 3 meses após a cirurgia, assim como observado em outro estudo $^{14}$. Nesse estudo, os autores avaliaram 28 mulheres antes e 30 dias após cirurgia de mama e também não encontraram modificações significantes na postura corporal nesse período. Porém, ressaltaram que, individualmente, todas as mulheres do estudo apresentaram modificações posturais e reforçam a importância do cuidado postural individualizado, a fim de evitar alterações futuras na biomecânica postural.

Entretanto, alguns estudos descrevem alterações posturais em mulheres submetidas ao tratamento por câncer de mama a longo prazo. Malicka et al. ${ }^{10}$ utilizando a mesma técnica, compararam a postura corporal de mulheres saudáveis e mulheres submetidas à mastectomia e quadrantectomia seis anos e meio após o tratamento e encontraram alterações nas curvaturas da coluna, sendo a postura considerada ruim em $82,3 \%$ das mulheres após tratamento de câncer de mama e em apenas 35,1\% das mulheres saudáveis avaliadas. Os autores observaram que mulheres após tratamento por câncer de mama apresentaram curva da cifose torácica mais acentuada e, $80 \%$ delas, relataram dor na coluna.

A queixa de dor na coluna foi mais frequente antes da cirurgia $(61,5 \%)$ do que após 3 meses $(25,6 \%)$. A prevalência de dor nas costas é maior em mulheres após 40 anos e apresenta relação com as atividades ocupacionais ${ }^{15,16}$. A média de idade das mulheres deste estudo foi de 50 anos e a atividade do lar foi a mais relatada pelas mulheres, seguida de doméstica e agricultora. Essas atividades podem sobrecarregar a coluna ocasionando alterações e algias. As mulheres deste estudo receberam orientações no Programa de Reabilitação de Mulheres Mastectomizadas do HSRC para evitar atividades domésticas e de sobrecarga de peso nos primeiros meses após a cirurgia. Acredita-se que ao acatar essas orientações, as mulheres reduziram o nível de atividades, podendo ser esse o motivo da redução de dor nas costas, já que nenhuma delas fazia uso de medicamentos analgésicos para esse fim.
Em outro estudo de seguimento, foram acompanhadas por dois anos mulheres após cirurgia por câncer de mama, a fim de identificar alterações posturais. Os autores compararam 2 tipos de cirurgia: mastectomia e reconstrução imediata, avaliando a postura corporal antes da cirurgia, 6 , 12,18 e 24 meses após a cirurgia. O seguimento mostrou que mulheres submetidas à reconstrução imediata tiveram menos prejuízos posturais após 24 meses do tratamento. Seis meses depois da cirurgia, a elevação e protrusão do ombro, associadas à rotação escapular do mesmo lado da cirurgia, estavam evidenciadas em mulheres mastectomizadas o que se manteve até o $24^{\circ}$ mês?

Ao correlacionar mastectomia e quadrantectomia com os ângulos posturais estudados, observou-se que a pelve e o tronco das mulheres submetidas à quadrantectomia estavam mais alinhados em relação àquelas submetidas à mastectomia.

Espera-se que, em cirurgias conservadoras, as alterações posturais sejam menores, pois não existe o importante fator mecânico da retirada da mama, e essas alterações ocorrem mais pelo aspecto psicológico, sensação de mutilação e dor ${ }^{7,9}$. Entretanto, não foram encontrados estudos que correlacionassem alterações posturais com as cirurgias em questão.

A maior parte das mulheres deste estudo foi submetida à cirurgia na mama esquerda $(61,5 \%)$. Ao correlacionar o lado da cirurgia com os ângulos medidos, o NO antes da cirurgia e no PO1, e a DT antes da cirurgia, no PO1 e no $\mathrm{PO} 2$, apresentaram valores significantes. Nas mulheres submetidas à cirurgia na mama esquerda, o ombro esquerdo apresentou-se mais elevado quando comparado àquelas que realizaram cirurgia do lado direito. Esse achado confirma os achados do estudo de Ciesla e Polom ${ }^{9}$, no qual seis meses após a mastectomia, as mulheres apresentavam elevação e protrusão do ombro, associadas à rotação escapular do lado homolateral à cirurgia. A DT que avalia a inclinação lateral do tronco demonstrou uma inclinação do tronco para o mesmo lado da cirurgia. Rostkowska, Bak e Samborski ${ }^{7}$ avaliaram e compararam a postura corporal de mulheres após câncer de mama e mulheres saudáveis, encontrando pior postura corporal nas mastectomizadas. Após três anos da cirurgia, essas apresentavam maior ângulo de inclinação anterior e lateral do tronco, maior valor dos ângulos das curvaturas vertebrais e ombros e escápulas assimétricos. Porém, a inclinação do tronco não apresentou correlação com o lado da cirurgia, mas sim com a idade. Mulheres mais velhas apresentavam uma inclinação mais frequente para a direita. No presente estudo, quando correlacionados os ângulos medidos e a DT com a idade da mulher não foram encontrados resultados significantes, sugerindo que todas as mulheres em tratamento precisam de cuidados preventivos em relação às alterações posturais independente da idade. 
A postura corporal de mulheres em tratamento e após o tratamento por câncer de mama apresenta modificações principalmente nas cirurgias de mastectomia e a longo prazo como foi discutido. De acordo com a análise dos dados deste estudo, observa-se que a postura corporal de mulheres com câncer de mama pode sofrer diferentes alterações no início e durante o tratamento. Porém, o número da amostra pode ter sido uma limitação para identificar outras alterações na postura, já que essas parecem ser sutis logo após a cirurgia, sendo tipicamente compensatórias e adaptativas. Torna-se necessário também, o seguimento do grupo após o término do tratamento, a fim de identificar a persistência das alterações posturais ou aparecimento de um padrão postural típico principalmente nas mulheres mastectomizadas, nas quais a retirada completa da mama é um fator mecânico importante a ser considerado quando se trata de biomecânica postural.

\section{Referências}

1. Schmidt MI, Duncan BB, Silva GA, Menezes AM, Monteiro CA, Barreto SM, et al. Doenças crônicas não transmissíveis no Brasil: carga e desafios atuais. The Lancet: Sáude no Brasil. 201 1;4:61-74.

2. Brasil. Ministério da Saúde. INCA [Internet]. Instituto Nacional de Câncer. Estimativa 2012: incidência de câncer no Brasil. Rio de Janeiro (R): INCA; 2011 . [citado 2013 Maio 17]. Disponível em: <http://bvsms.saude.gov.br/bvs/controle_cancer>

3. Brasil. Ministério da Saúde. Instituto Nacional de Câncer. Controle do câncer de mama: documento de consenso. Rev Bras Cancerol. 2004;50(2):77-90.

4. Almeida AM, Prado MAS, Guidorizzi LLF, Rossini FP. Mulheres com câncer de mama: um estudo de morbidade. Acta Oncol Bras. 2002;22(2):263-9.

5. Rietman JS, Dijkstra PU, Hoekstra HJ, Eisma WH, Szabo BG, Groothoff JW, et al. Late morbidity after treatment of breast cancer in relation to daily activities and quality of life: a systematic review. Eur J Surg Oncol. 2003;29(3):229-38.

6. Melo MSI, Maia JN, Silva DAL, Carvalho CC. Avaliação postural em pacientes submetidas à mastectomia radical modificada por meio da fotogrametria computadorizada. Rev Bras Cancerol. $2011 ; 57(1): 39-48$.

7. Rostkowska E, Bak M, Samborski W. Body posture in women after mastectomy and its changes as a result of rehabilitation. Adv Med Sci. 2006;51:287-97.

8. Nascimento SL, Oliveira RR, Oliveira MMF, Amaral MTP. Complicações e condutas fisioterapêuticas após cirurgia por câncer de mama: estudo retrospectivo. Fisioter Pesqui. 2012;19(3):248-55.
9. Ciesla S, Polom K. The effect immediate breast reconstruction with Becker-25 prosthesis on the preservation of proper body posture in patients after mastectomy. Eur J Surg Oncol. 2010;36(7):625-31.

10. Malicka I, Barczyk K, Hanuszkiewicz J, Skolimowska B, Woźniewski M. Body posture of women after breast cancer treatment. Ortop Traumatol Rehabil. 2010;12(4):353-361.

11. Ricieri DV, Costa JR, Rosário Filho NA. Impacto da asma sobre a postura corporal de crianças entre 8 e 14 anos analisada pela biofotogrametria. Acta Fisiátrica. 2008; 15(4):214-9.

12. lunes DH, Bevilaqua-Grossi D, Oliveira AS, Castro FA, Salgado HS. Análise comparativa entre avaliação postural visual e por fotogrametria computadorizada. Rev Bras Fisioter. 2009; 13(4):308-15.

13. Perin A, Ulbricht L, Ricieri DV, Neves EB. Utilização da biofotogrametria para a avaliação da flexibilidade de tronco. Rev Bras Med Esporte. 2012; 18(3):176-80.

14. Bregagnol RK, Dias AS. Alterações funcionais em mulheres submetidas à cirurgia de mama com linfadenectomia axilar total. Rev Bras Cancerol. 2010;56(1):25-33.

15. Ferreira GD, Silva MC, Rombaldi A, Wrege ED, Siqueira FV, Hallal PC. Prevalência de dor nas costas e fatores associados em adultos do Sul do Brasil: estudo de base populacional. Rev Bras Fisioter. 2011;15(1):31-6.

16. Matos MG, Hennington EA, Hoefel AL, Dias-da-Costa JS. Dor lombar em usuários de um plano de saúde: prevalência e fatores associados. Cad Saúde Pública. 2008;24(9):21 15-22. 